\title{
A SPECIFIC NANOMANUFACTURING CHALLENGE
}

\author{
M J Kelly ${ }^{1,2,3 *}$ and M C Dean ${ }^{1,2}$
}

${ }^{1}$ Cavendish Laboratory, Department of Physics, University of Cambridge, J. J. Thomson

Avenue, Cambridge, CB3 OHE, United Kingdom 6

${ }^{2}$ Centre for Advanced Photonics and Electronics, Electrical Engineering Division, Department

of Engineering, $9 \mathrm{~J}$. J. Thomson Avenue,

University of Cambridge, Cambridge CB3 OFA, United Kingdom

${ }^{3}$ The MacDiarmid Institute, Victoria University of Wellington, Wellington 6140, New Zealand

Abstract: For a science to become a technology, a certain level of control has to have been established over the way items are fabricated for manufacture and use. Here we first consider the challenge of making and using a LEGO ${ }^{\circledR}$ brick scaled down by a factor of $10^{n}$ for $n=0-6$ in each spatial dimension, i.e. from millimetres to nanometres. We consider both the manufacture and the subsequent properties of the nanobricks that pertain to their use in constructing and dismantling structures. As $n$ increases, the ability to use fails first, to manufacture fails second and to fabricate fails last. Applied to the vast literature in nanoscience, this process emphasises the unmanufacturability of most nanoscale artefacts.

Much of what passes today for nanotechnology is still in fact nanoscience. Technology implies a level of control or engineer-ability over the fabrication and use of artefacts. When it comes to manufacturing artefacts, we need to be able to reproduce them by the thousands/millions, having them meet a prior specification of performance to a given tolerance with a sufficiently small number of defects. For example in modern integrated circuit manufacture, one achieves six-sigma quality, with only a few transistors in a million out of specification at something like the $10 \%$ tolerance level for a threshold voltage. Again one approaches say six-sigma (or five-nines purity in molecular terms) for very few non-trivial molecules of a few nanometres spatial extent. In modern electronics, it would appear that circuits based on a $5-7 \mathrm{~nm}$ half-pitch border on being unmanufacturable (in terms of high and reproducible yield to tight tolerances at the six-sigma level) [1]. Indeed whereas Feynman gave rise to nanoscience with his lecture 'There is plenty of room at the bottom', there is not so much room at the bottom of nanotechnology, with its implication of manufacturability and utility.

In order to explore the manufacturability of a specific nanoscale artefact we will choose a somewhat arbitrary, but instructive, example: a nanoscale model of the Eiffel Tower constructed entirely out of nanoscale Lego ${ }^{\circledR}$, or nanobricks. We will begin by considering the requirements on our nanobricks mechanically and the implications this has for tolerances in the manufacturing process. Top down and bottom up manufacturing techniques will then be considered with the goal of determining the smallest working brick that could be made and used. 
The yellow brick in Figure 1 has dimensions of $7.8 \mathrm{~mm} \times 7.8 \mathrm{~mm} \times 9.6 \mathrm{~mm}$, and the tolerance of the feature sizes is of order $10 \mu \mathrm{m}$, or typically $1 / 1000$, which allows successive bricks to combine with sufficient rigidity that a stable 100-high brick tower can be constructed.

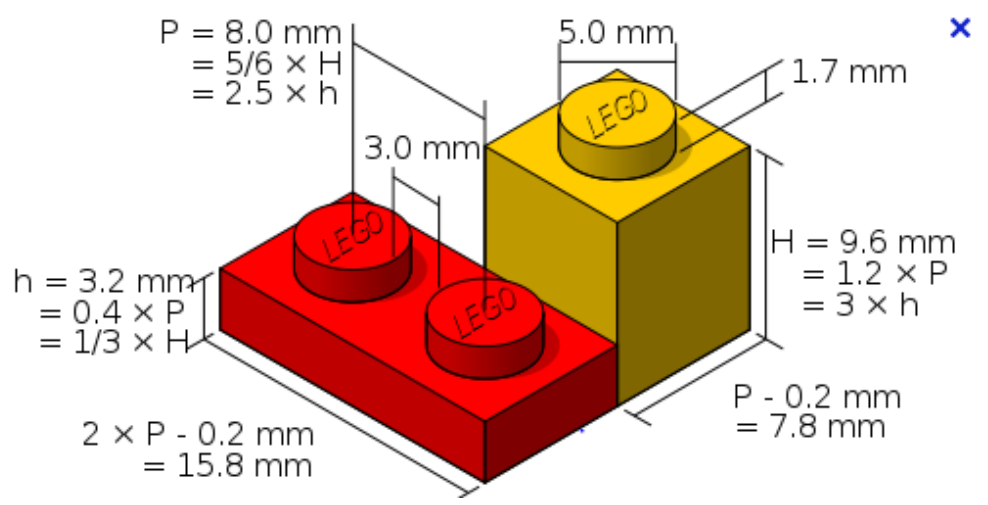

Figure 1: Dimensions of standard Lego ${ }^{\circledR}$ bricks. We will assume a simplified underside where just the hole to receive the cylinders is retained, leaving the brick otherwise solid rather than hollow as in conventional Lego ${ }^{\circledast}$. A brick with a skin of only a few nanometres thickness would be too fragile. See http://www.lucasbrouwers.nl/blog/2011/05/why-life-is-like-lego/

We now consider what happens as we shrink each side by successive factors of 10 , and the results are summarised in Table 1, considering (i) the technology used to fabricate (on a one-off basis), (ii) the technology to manufacture (in thousands/millions) and (iii) the tools used to build and deconstruct an Eiffel tower, and the ease of use of these tools. As the scale down proceeds, the ability to use fails first, to manufacture fails second and to fabricate fails last. The use fails for several reasons, one being the tolerance at $1 / 1000$ is less easily scaled, and the inability to grow a stable tower 100 bricks high at the $1 \mu \mathrm{m}$ scale. Note that there are at least two technologies (i) imprint stamping and (ii) deposition, lithography and etching that persist as fabrication technologies down to the nanoscale. There are many further aspects that can be considered instructively at each scale: yield, reproducibility, wear and tear, etc.

Table 1: Milli- Micro- and Nano-Bricks.

\begin{tabular}{|l|l|l|l|l|}
\hline Scale ${ }^{*}$ & Fabricate $\neq$ & Manufacture $\neq$ & Can Use/User Tool & Comment on use \\
\hline $1 \quad \mathrm{~mm}$ & $\mathrm{IM}(\mathrm{p})$ & $\mathrm{IM}(\mathrm{p})$ & Yes/Hands & Easy \\
\hline $10^{-1}$ & $\mathrm{IM}(\mathrm{p})$ & $\mathrm{IM}(\mathrm{p})$ & Yes/Tweezers & Straightforward \\
\hline $10^{-2}$ & $\mathrm{IS}(\mathrm{p})$ & $\mathrm{IS}(\mathrm{p})$ & Yes/Micro-tweezers & Tricky \\
\hline $10^{-3} 1 \mu \mathrm{m}$ & $\mathrm{IS}(\mathrm{p}), \mathrm{DLE}(\mathrm{s})$ & $\mathrm{IS}(\mathrm{p}), \mathrm{DLE}(\mathrm{s})$ & Yes/Micro-tweezers, AFM & Demanding \\
\hline $10^{-4}$ & $\mathrm{IS}(\mathrm{p}), \mathrm{DLE}(\mathrm{s})$ & $\mathrm{IS}(\mathrm{p}), \mathrm{DLE}(\mathrm{s})$ & Yes/AFM & Only at relaxed tolerances. \\
\hline $10^{-5}$ & $\mathrm{IS}(\mathrm{p}), \mathrm{DLE}(\mathrm{s})$ & No & No & Impossible \\
\hline $10^{-6} 1 \mathrm{~nm}$ & AFM & No & No & Impossible \\
\hline
\end{tabular}

* The scale is set in comparison with real LEGO bricks that are on the scale of $8 \mathrm{~mm}$ on a short side and $3-5 \mathrm{~mm}$ in terms of the top pillar on the brick.

₹ Key: $\mathrm{IM}(\mathrm{p})$ = injection molding of plastic; $\mathrm{IS}(\mathrm{p})=$ Imprint stamping of plastic; $\mathrm{DLE}(\mathrm{s})=$ Deposition, lithography, etch (semiconductor) 
The challenge of manufacturing 1000 Nanoscale LEGO $^{\circledR}$ Bricks, and using them to construct and deconstruct a small scale Eiffel Tower is one which will help define in practical terms when a nanoscience idea has truly become a nanotechnology, in the sense in which technology is usually meant, namely the ability to make many pieces all the same within a narrow pre-specification and tolerance to perform a pre-specified function. We commend this specific challenge as a useful comparator to those developing proposed nanotechnologies to establish a clear and generic proof that the technology is available. Note that we have considered individual artefacts as the manufactured item. In some applications, it is the aggregate properties of many such artefacts that are exploited: in this case the manufacturability is achieved when the average and standard deviation of the size of the individual artefacts is under the same high level of control to be able to meet pre-specified values with an acceptable tolerance.

Acknowledgements: Acknowledgements: We thank EPSRC for support under grant EP/J010888/1.

[1] Nedelcu M, Saifullah M S M, Hasko D G, Jang A, Anderson D, Huck W T S, Jones G A C, Welland M E, Kang D J and Steiner U 2010 'Nanowires: fabrication of Sub-10 nm metallic lines of low line-width roughness by hydrogen reduction of patterned metal-organic materials', Adv. Funct. Mater. 20 2317-23

[2] [1] M J Kelly, 'Intrinsic top-down unmanufacturability' Nanotechnology 22 (2011) 245303 